\title{
Amenagement des produits forestiers ligneux et non ligneux dans la region de 1'Est-Cameroun : Cas de la station polyvalente de recherche agricole d'Abong Mbang
}

\section{BAYOI Emmanuel Arme1 ${ }^{1,4}$, MENYENE ETOUNDI Florent ${ }^{1,2,3}$, MOUYAKAN A MOUMBOCK Elvis ${ }^{1,2,4}$, Mbezele Junior Yannick NGABA ${ }^{5,6 *}$}

${ }^{1}$ Ministère de la Recherche Scientifique et de l'Innovation (MINRESI), Yaoundé, Cameroun

${ }^{2}$ Département de Biologie des organismes végétaux, Faculté des Sciences (FS), Université de Yaoundé 1, BP 337 Yaoundé Cameroun.

${ }^{3}$ Département de Biologie des organismes végétaux, Faculté des Sciences (FS), Université de Douala, BP 8580 Douala Cameroun.

${ }_{4}^{4}$ Institut de Recherche Agricole pour le Developpement (IRAD), Nkolbisson, BP 2123 Yaounde, Cameroun

${ }^{5}$ College of Natural Resources and Environment, Northwest A\&F University, Yangling 712100, Shaanxi, China

${ }^{6}$ Key Laboratory of Plant Nutrition and the Agri-Environment in Northwest China, Ministry of Agriculture, Yangling 712100, Shaanxi, China

*Corresponding author: Dr Mbezele Junior Yannick NGABA

\section{Résume}

Les produits forestiers ligneux (PFL) et non ligneux (PFNL) jouent un rôle important pour le bien-être des populations au Cameroun. Malheureusement, elles font face à plusieurs menaces telle que l'exploitation commerciale de la ressource et l'exploitation anarchique de bois d'œuvre ; l'absence ou l'insuffisance de donnée scientifiques nécessaires pour les prises de décision d'aménagement. L'objectif de l'étude est d'identifier et d'évaluer la disponibilité des espèces végétales dans la forêt de la station polyvalente de recherche agricole d'Abong Mbang. Un inventaire d'aménagement a été effectué avec un taux de sondage de 5\%. Il ressort de cette étude qu'il y une extrême diversité des PFL et PFNL dans la zone d'étude. Un total de 69 PFL (bois d'œuvre) et 18 PFNL sont utilisées dans la localité pour l'alimentation, la santé ou l'ornement. Les fruits et les feuilles sont généralement utilisés pour la consommation alors que les écorces sont à usage médicinale. Les connaissances ancestrales des populations locales sur cette ressource est approfondie et contribue à leur bien-être ont une des PFNL.

Mots clés : PFNL, espèces de bois, aménagement forestier, Abong Mbang, Est-Cameroun.

Received: 01/06/2021

Accepted: 09/08/2021

DOI: https://dx.doi.org/10.4314/jcas.v17i1.1

(C) The Authors. This work is published under the Creative Commons Attribution 4.0 International Licence. 


\begin{abstract}
Timber and non-timber forest products (NTFPs) play an important role for the well-being of the populations in Cameroon. Unfortunately, they face several threats such as the commercial exploitation of the resource and the uncontrolled exploitation of timber; the lack or insufficiency of scientific data necessary for management decisions. The objective of the study is to identify and evaluate the availability of plant species in the forest of the Abong-Mbang Agricultural Research Station. A management inventory was conducted with a sampling rate of $5 \%$. The study revealed that there is an extreme diversity of LFAs and NTFPs in the study area. A total of 69 LFWPs (timber) and 18 NTFPs are used in the locality for food, health or ornamental purposes. Fruits and leaves are generally used for consumption while barks are used for medicinal purposes. The ancestral knowledge of local populations on this resource is deep and contributes to their well-being have a NTFP.
\end{abstract}

Keywords: NTFPs, wood species, forest management, Abong Mbang, East Cameroon. 


\section{Introduction}

Les forêts du Cameroun regorgent une diversité floristique importante et variée qui couvrent environ $42 \%$ de sa superficie (FAO, 2011), et jouent un rôle crucial pour le gouvernement et les populations du Cameroun. En effet, l'exploitation forestière communément appelé 6 l'or vert3 contribue de façon significative au produit intérieur brut (PIB) de l'économie nationale Camerounaise. L'exploitation des produits forestiers ligneux (PFL) ou bois d'œuvre industriels des forêts naturelles par exemple, rapporte des bénéfices estimés à 368,3 milliards (Eba et al., 2013). Les produits forestiers non ligneux (PFNL) contribuent à près de 64,12 milliards d'après le même auteur. Toutefois, ces ressources font l'objet de fortes pressions dues à l'exploitation abusive et non contrôlée (Ngaba et al., 2019; Olivier et al., 2020). Ajouté à cela il existe un vide règlementaire qui ne clarifie pas les dispositions à prendre pour les populations locales après l'attribution d'une concession forestière aux exploitants forestiers. Créant de ce fait, une double problématique de 6 profitabilité3 et 6 d'équitabilité 3 entre le gouvernement Camerounais, les exploitants forestiers et les populations riveraines qui pourtant sont protégées par le droit d'usage ou droit coutumier (Moumbock et al., 2020; Topa et al., 2009). Les PFNL participent significativement à la lutte contre la pauvreté de ces populations comme source de revenu via la commercialisation et requièrent des valeurs alimentaires, thérapeutiques et socio-culturelles indéniables (Ngijol et al., 2020; Tchatat and Ndoye, 2006).

La collecte des PFNL demeure une activité très largement pratiquée au Cameroun (Lescuyer, 2010). En vue de garantir l'utilisation durable de ces ressources forestières, plusieurs mesures ont été mise en place à l'instar de la loi forestière $n^{\circ} 94 /$ 01 du 20 janvier 1994 portant régime des forêts, de la faune et de la pêche. Couplé à plusieurs décrets et textes d'application notamment le décret $\mathrm{n}^{\circ}$ 95/531/PM du 23 août 1995 fixant les modalités d'application du régime des forêts. L'intérêt du Cameroun pour la protection des PFL et PFNL est également marqué par la Décision $n^{\circ}$ 2032/D/MINFOF du 22 août 2012 fixant la liste des produits forestiers spéciaux et le programme sectoriel forêt environnement (PSFE) qui prônent la valorisation durable des PFNL.

On peut également citer entre autres l'amélioration des connaissances du patrimoine forestier par la mise en place des stations de recherche dont les résultats permettront de guider les décisions de gestion. L'Institut camerounais de recherche agricole pour le développement (IRAD) dans sa politique de recherche forestière et environnementale a initié dans le cadre du Budget d'Investissement Public (BIP) plusieurs études en vue de favoriser la reconstitution des peuplements naturels de bois et PFNL après exploitation en zone de forêt dense, savane humide et sèche du Cameroun. Il a par ailleurs comme attribution l'évaluation du potentiel de bois d'œuvre et des PFNL au Cameroun. C'est ainsi que la Station de Valorisation Agricole (SVRA) a été créée en 2020 pour la programmation et de l'exécution des programmes et projets de recherche et de développement. Toutefois, aucune étude n'a encore été réalisée dans cet écosystème forestier pouvant renseigner sur son potentiel en bois d'œuvre sur pieds et PFNL. La présente étude vise à déterminer la typologie et l'importance des PFNL pour les populations riveraines, évaluer le potentiel ligneux des essences de bois dans la zone d'étude.

\section{Méthodologie \\ Présentation du site d'étude}

Le village Oboul se trouve en plein cœur de la forêt tropicale humide sempervirente du Bassin du Congo (CNUCED, 2008). Précisément dans l'Arrondissement d'Abong-Mbang, le 


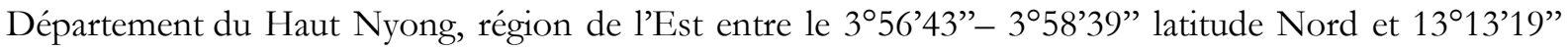
- 1314’30” longitudes Est (Figure 1). Elle couvre une superficie de 121,42 ha.
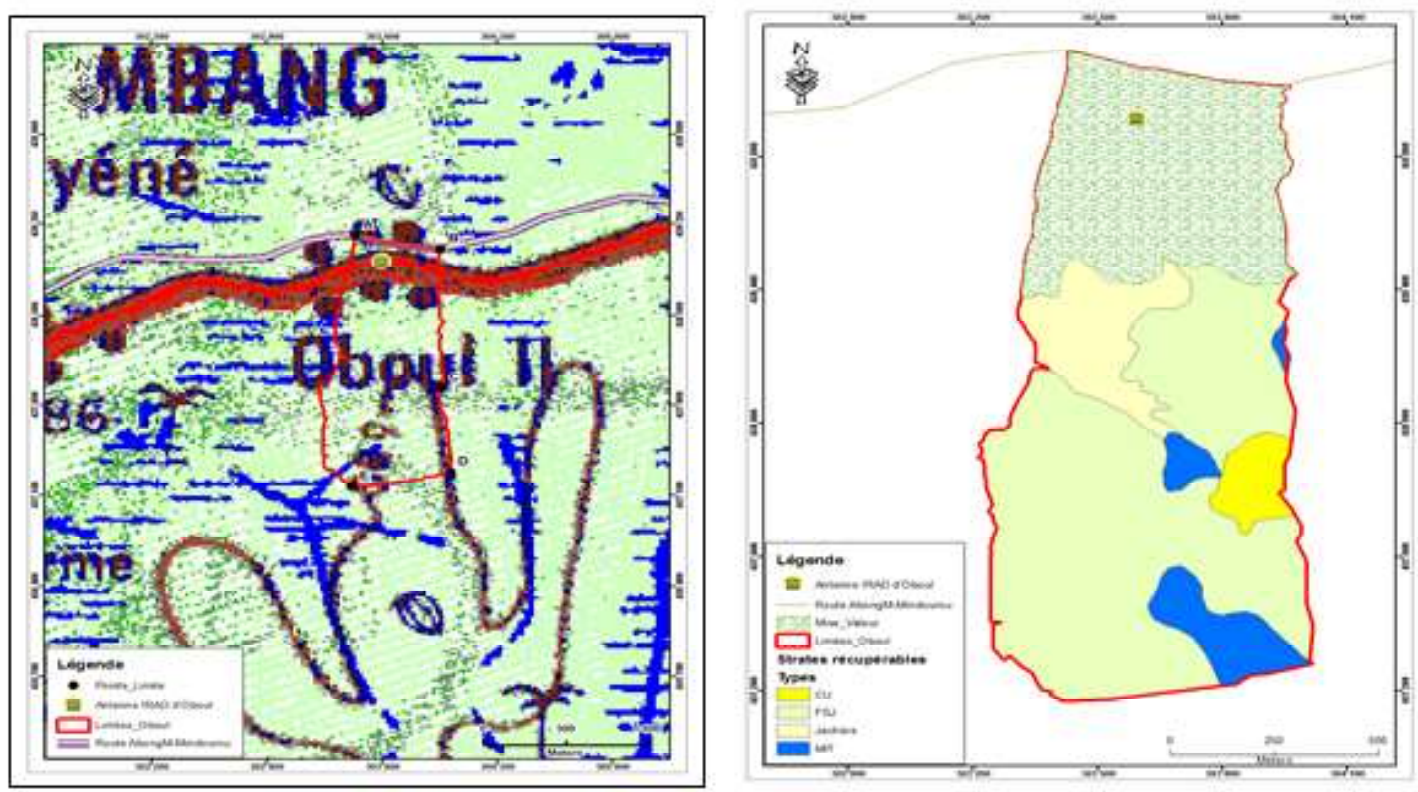

Figure 1. Carte forestière de l'assiette IRAD Oboul

CU : culture ; FSJ : Forêt Secondaire Jeune ; MIT : Marécage Inondé Temporairement.

\section{Description de l'assiette IRAD Oboul}

Le point de base A des limites de l'IRAD d'Oboul a pour coordonnées : UTM : $\mathrm{X}_{(\mathrm{m})}=303438 ; \mathrm{Y}_{(\mathrm{m})}=$ 439 112. Le périmètre de la Figure 2 passe par les points : A, B, C et D dont les coordonnes UTM sont présentées dans le Tableau 1.

Tableau 1. Coordonnés des points de bornage des limites

\begin{tabular}{llllll}
\hline Nom & A & B & C & D & E \\
\hline POINT_X & 303438 & 303958 & 303964 & 304018 & 303416 \\
POINT_Y & 439112 & 439013 & 437933 & 437280 & 437169 \\
\hline
\end{tabular}

\section{Collecte des données}

Les données secondaires ont été collectées à travers la revue de littérature (rapports, livres, articles scientifiques ou mémoires de fin de formation). Les données primaires ont été collectées lors des descentes de terrain. La collecte de données sur les PFNL s'est faite à travers des enquêtes semi-structurées auprès de personnes directement ou indirectement concernées par la gestion des PFNL (les ménages ou membres de GIC). Les informations reçues ont permis de faire une caractérisation des PFNL existants dans la zone, leur utilité et usage. Les cinq premiers mètres parcourus sur le layon (parcelle floristique) permettent de recenser premièrement les Gaulis dont les diamètres sont compris entre $[10-20 \mathrm{~cm}]$ alors que les tiges dont le diamètre est supérieur ou égal à $20 \mathrm{~cm}$ sont inventoriés sur toute la longueur de la placette. Les layons ont été subdivisés en placette de 250 m. Ce qui a permis d'identifier et de confirmer les différentes strates présentées dans la carte forestière de ce massif (Tableau 2). Le Tableau 3 présente les coordonnées géographiques des points de départ et d'arrivé de chaque layon de comptage du dispositif de sondage et précise la distance et azimut (angle) réelle à parcourir. La longueur totale des layons à parcourir est de 2,087 $\mathrm{km}$; ce qui permettra de maintenir un taux de sondage au-dessus du minimum de $1 \%$ requis, le provisionnel étant fixé à 5\%. A l'intérieur de chaque unité de comptage, le sens du comptage a été pris en compte et celui des virés à réaliser entre deux layons consécutifs comme l'illustre la Figure 2 du plan de sondage. 
Tableau 2. Données techniques du plan de sondage de l'assiette IRAD Oboul

\begin{tabular}{ll}
\hline Paramètres de sondage & Valeurs \\
\hline Superficie totale & $85 \mathrm{ha}$ \\
Taux de sondage & $5 \%$ \\
Superficie sondée & $4,174 \mathrm{ha}$ \\
Superficie placette & $0,5 \mathrm{ha}$ \\
Nombre de placettes & 8,348 \\
Longueur totale des layons & $2,087 \mathrm{~km}$ \\
Equidistance & $350 \mathrm{~m}$ \\
\hline
\end{tabular}

Tableau 3. Coordonnées départs et arrivées des layons de comptage

\begin{tabular}{|c|c|c|c|c|c|c|}
\hline \multirow{2}{*}{ Layons } & \multicolumn{2}{|c|}{ Coord Départ } & \multicolumn{2}{|c|}{ Coord Arrivées } & \multirow{2}{*}{$\begin{array}{l}\text { Longueur } \\
\text { (m) }\end{array}$} & \multirow{2}{*}{ GIS $\left(^{\circ}\right)$} \\
\hline & Point_X & Point_Y & Point_X & Point_Y & & \\
\hline$\overline{\mathrm{LC}-01}$ & 303954 & 438219 & 303349 & 438219 & 604 & 270 \\
\hline LC-02 & 303948 & 437869 & 303227 & 437869 & 721 & 270 \\
\hline LC-03 & 304003 & 437519 & 303241 & 437519 & 762 & 270 \\
\hline TOTAL & & & & & 2087 & \\
\hline
\end{tabular}

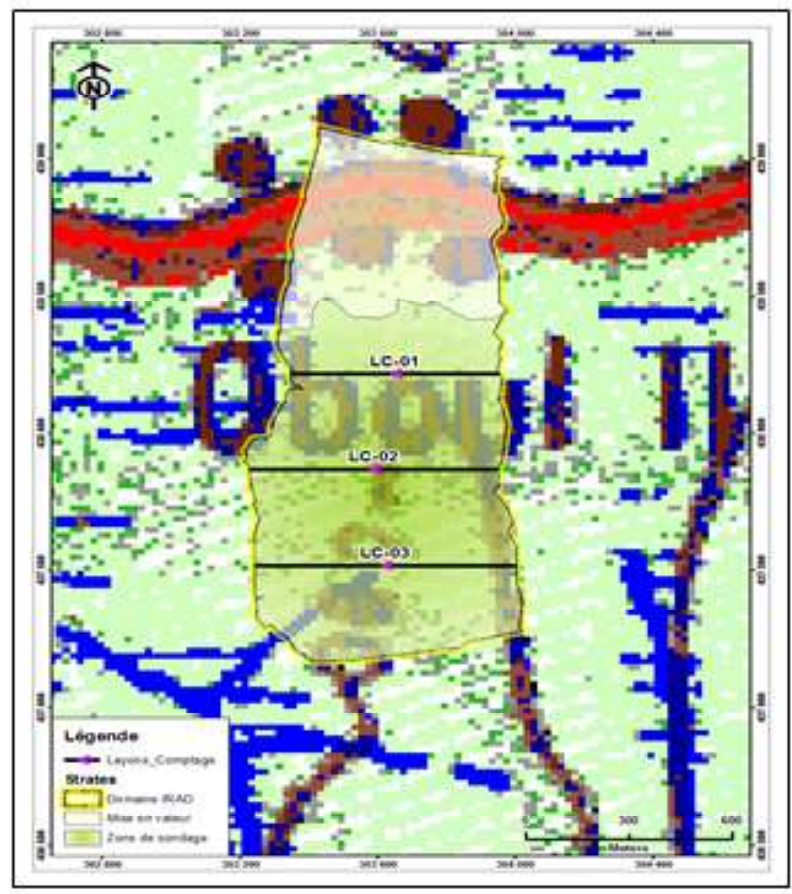

Figure 2. Plan de sondage de l'assiette IRAD Oboul 


\section{Analyse des données d'inventaire}

Les données ont été encodées, triées dans le logiciel Microsoft Excel 2016. Il a par ailleurs servi

à la réalisation des tableaux (densité, volume, surfaces terrières à l'hectare ou totaux). Le logiciel Origine pro version 2021 et TIAMA quant à eux ont permis d'encoder et analyser les données de terrain collectées et produire les tableaux et diagrammes. Les cartes ont été produites grâce au logiciel ArcGIS 10.3. Il a également servi a l'élaboration des cartes de répartition de la ressource (en effectifs, volumes ou surface terrière). Les calculs d'erreurs statistiques ont été effectués automatiquement et permettent d'apprécier la qualité des résultats obtenus avant d'en effectuer l'interprétation.

\section{Résultats et discussions}

\section{Evaluation du potentiel ligneux}

Essences rencontrées et contenance de la forêt

La forêt étudiée présente une richesse floristique représentative de la biodiversité des forêts de la zone de l'Est Cameroun. Le Tableau 4 présente toutes les essences forestières exploitables présentes dans la forêt de recherche de la station de valorisation Agricole IRAD à Abong Mbang. Le Tableau 5 quant à lui illustre sur la base des travaux de prospection, layonnage et comptage, la contenance de la forêt soumise à notre étude décrite sur la base du type de terres ou strate retrouvée sur le site à inventorier. Le Tableau 5 présente la contenance de la forêt étudiée essentiellement regroupée en deux écosystèmes soient de terres forestières $(83.13 \%)$ et de terres non boisées $(16.87 \%)$ avec pour superficies respectives 71.41 ha et 14.49 ha.

\section{Distribution des tiges et courbe de distribution}

Le Tableau 6 présente la distribution des essences repartie par classe de diamètre et par type de strate. Les strates retrouvées sont : les forêts secondaires jeunes (SJ), les zones agroforestières (AGF), les marécages inondés temporairement (MIT), les zones de cultures (CU) et les autres strates (AT). Ce qui démontre que l'utilisation des PFNL à une valeur culturel (Zima et al., 2018). Il présente la superficie distribuée dans chacune des strates, les classes de diamètre et le volume réel existent dans chacune des strates.

Il ressort de la Figure 3 que, la classe de diamètre ayant la plus forte densité varie de 20 à 60 centimètres. Ainsi on se rend compte d'une faible densité des arbres exploitables dans la zone ce qui justifie la strate dominante (FSJ) de la forêt et ouvre de nouvelle possibilité d'aménagement. Plusieurs essences exploitables ont des diamètres inférieurs au Diamètre Minimum d'Exploitabilité Administratif (DME / ADM) et gagneraient à être conservés et enfin suivi.

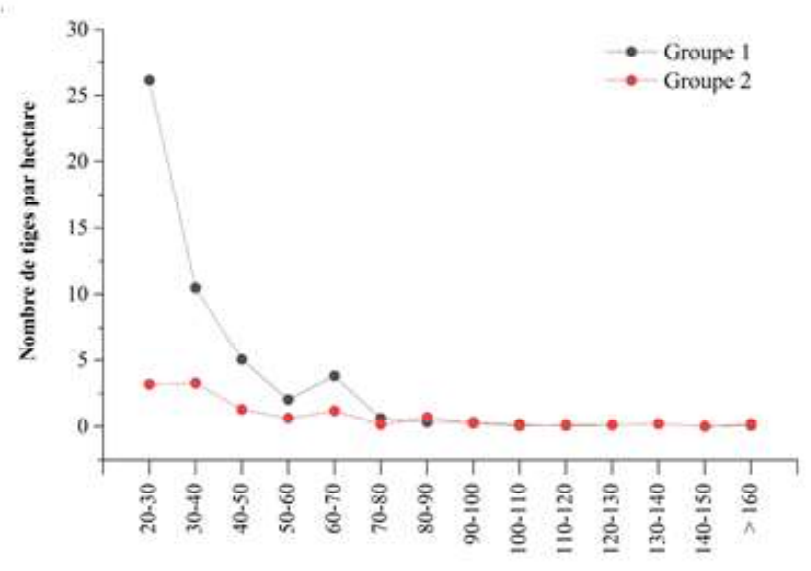

Figure 3. Courbe de distribution des tiges de l'assiette IRAD Oboul.

Groupe 1 : Essences plus denses et jeunes; Groupe 2 : Essences moins denses et jeunes 
Tableau 4. Groupes d'essences rencontrées dans l'assiette IRAD Oboul

\begin{tabular}{|c|c|c|}
\hline $\mathbf{N}^{\circ}$ & Noms commerciaux & Noms scientifiques \\
\hline 1 & Ayous / Obeche & Triplochyton scleroxylon \\
\hline 2 & Iroko & Milicia excels \\
\hline 3 & Kotibé & Nesogordonia papaverifera \\
\hline 4 & Aningré A & Aningeria altissima \\
\hline 5 & Bongo H (Olon) & Fagara heitzii \\
\hline 6 & Eyong & Eribroma oblongum \\
\hline 7 & Lotofa / Nkanang & Sterculia rhinopetala \\
\hline 8 & Movingui & Distemonanthus benthamianus \\
\hline 9 & Aiélé / Abel & Canarium schweinfurthii \\
\hline 10 & Alep & Desbordesia glaucescens \\
\hline 11 & Bilinga & Nauclea diderrichii \\
\hline 12 & Dabéma & Piptadeniastrum africanum \\
\hline 13 & Emien & Alstonia boonei \\
\hline 14 & Fraké / Limba & Terminalia superba \\
\hline 15 & Fromager / Ceiba & Ceiba pentandra \\
\hline 16 & Ilomba & Pycnanthus angolensis \\
\hline 17 & Padouk rouge & Pterocarpus soyauxii \\
\hline 18 & Tali & Erythropleum ivorense \\
\hline 19 & Diana Z & Celtis zenkeiri \\
\hline 20 & Ekouné & Coelocaryon preussi \\
\hline 21 & Iantandza & Albizia ferruginea \\
\hline 22 & Kumbi & Lannea welwitschii \\
\hline 23 & Landa & Erythroxylum mannii \\
\hline 24 & Lati parallèle & Amphimas pterocarpoides \\
\hline 25 & Mutondo & Funtumia elastic \\
\hline 26 & Abalé & Petersianthus macrocarpus \\
\hline 27 & Abena & Homalium letestui \\
\hline 28 & Akui & Xylopia aethiopica \\
\hline 29 & Angakomo & Barteria fistulosa \\
\hline 30 & Angoan & Porterandia cladantha \\
\hline 31 & Angossa & Markhamia tomentosa \\
\hline 32 & Assas & Macaranga burifolia \\
\hline 33 & Ayinda & Anthocleista schweinfurtbii \\
\hline 34 & Bongo Jean Marie & Fagara lemairei \\
\hline 35 & Bongo nkol petites feuilles & Fagara dinklagei \\
\hline 36 & Diana $T$ & Celtis tessmannii \\
\hline 37 & Ebai & Pentacletbra eetveldeana \\
\hline 38 & Ebegbemva osoé & Trichilia welwitschii \\
\hline 39 & Ebom & Anonidium mannii \\
\hline 40 & Efobolo & Tetrorchidium didymostemon \\
\hline 41 & Efok afum / Poré poré & Sterculia tragacantha \\
\hline
\end{tabular}


REVUE DE L'ACADEMIE DES SCIENCES DU CAMEROUN Vol. 17 No. 1 (Octobre 2021)

\begin{tabular}{|c|c|c|}
\hline 42 & Enga am & Ormocarpum bibracteanum \\
\hline 43 & Engokom & Myrianthus arboreus \\
\hline 44 & Essak / Alow kouaka & Albizia glaberrima \\
\hline 45 & Essesang & Ricinodendron heudelotii \\
\hline 46 & Etoan & Tabernae montana crassa \\
\hline 47 & Kapokier & Bombax buonopozense \\
\hline 48 & Lo & Parkia bicolor \\
\hline 49 & Mbang mbazoa avié & Strombosia grandifolia \\
\hline 50 & Mebenga osoé & Barteria nigritiana \\
\hline 51 & Mfang mvanda & Gilletiodendron kisantuense \\
\hline 52 & Mféneg & Desplatsia dewevrei \\
\hline 53 & Minsi & Calpocalyx dinklagei \\
\hline 54 & Mvanda & Hylodendron gabonense \\
\hline 55 & Musizi & Maesopsis eminii \\
\hline 56 & Nkok élé & Polyscias fulva \\
\hline 57 & Nom akui & Xylopia bypolampra \\
\hline 58 & Nom miasmingomo & Caloncoba glauca \\
\hline 59 & Nom miasmingomo ahin & Caloncoba brevipes \\
\hline 60 & Obatoan & Tabernae montana pachysiphon \\
\hline 61 & Oloa & Lecaniodiscus cupanioides \\
\hline 62 & Osomzso & Bosqueia angolensis \\
\hline 63 & Оуо & Brenania brieyi \\
\hline 64 & Parasolier & Musanga cecropioides \\
\hline 65 & Saliyemo nkol yangba & Albizia gummifera \\
\hline 66 & Saliyemo / Bangbaye & Albizia adianthifolia \\
\hline 67 & Tol / Figuier & Ficus mucuso \\
\hline 68 & Mengamenjanga & Rauvolfia vomitoria \\
\hline 69 & Nom ebegbemva & Trichilia dregeana \\
\hline
\end{tabular}

Tableau 5. Table de contenance de l'assiette IRAD Oboul

\begin{tabular}{|c|c|c|c|c|c|}
\hline \multicolumn{6}{|l|}{ Terrains forestiers } \\
\hline & Strate & Affectation & Nb. & Superficie & Total \\
\hline Secondaire & SJ & FOR & 5 & 63,73 & 74,2 \\
\hline \multirow[t]{2}{*}{ Sol hydromorphe } & MIT & FOR & 1 & 7,68 & 8,94 \\
\hline & Sous-total : & 6 & 71,41 & 83,13 & \\
\hline \multicolumn{6}{|l|}{ Terrains non-boisés } \\
\hline & Strate & Affectation & Nb. & Superficie & Total \\
\hline Milieux agricoles & $\mathrm{CU}$ & AGF & 1 & 3,95 & 4,6 \\
\hline \multirow[t]{3}{*}{ Milieux urbanisés } & AT & AUT & 1 & 10,54 & 12,27 \\
\hline & Sous-total & 2 & 14,49 & 16,87 & \\
\hline & TOTAL & 8 & 85,89 & 100 & \\
\hline
\end{tabular}


Table de peuplement et de stock

Le comptage réalisé dans les placettes de chaque layon contenant chacune, une strate précise, a permis la mise en place d'une table de peuplement précisant le nom de l'essence, le code de l'essence et le nombre de tiges à l'hectare essence comme l'illustre le Tableau 6. Les résultats de l'analyse des données d'inventaire ont permis de savoir que la forêt aménagée compte précisément quinze (15) essences principales avec un total d'environ deux cent (200) pieds exploitables avec un DME supérieur ou égale au DME/ADM. Notons ainsi que la forêt étudiée est ainsi exploitable avec des essences très prisées comme : le Movingui (Distemonanthus benthamianus), le Padouk (Pterocarpus soyauxii); l'Iroko (Milicia excels); le
Fraké (Terminalia superba) et l'Aningré

(Aningeria altissima); capable à elles seules d'apporter un intérêt pour les sociétés d'exploitation dans la zone (FIPCAM, PALLISCO...). Mais aussi de susciter un intérêt sur le plan de la recherche.

Le Tableau 7 présente la table de stock de l'inventaire réalisé dans la forêt de station de valorisation agricole d'Abong Mbang. Il ressort du Tableau 7 que la forêt aménagée compte précisément quinze (15) essences principales avec un volume total disponible d'environ mille sept cent mètre cubes $\left(1700 \mathrm{~m}^{3}\right)$ dont $978 \mathrm{~m}^{3}$ exploitables avec un DME supérieur ou égale au DME/ADM

Tableau 6. Table de peuplement de l'assiette IRAD Oboul

\begin{tabular}{llllll}
\hline Essence & Nom scientifique & Code & Tiges/ha & Tiges total & Tiges $\geq$ DME \\
\hline Aiélé/Abel & Canarium schweinfurthii & 1301 & 0,36 & 25 & 0 \\
Aningré A & Aningeria altissima & 1201 & 0,36 & 25 & 25 \\
Ayous/Obeche & Triplochyton scleroxylon & 1105 & 0,21 & 15 & 15 \\
Bongo H (Olon) & Fagara heitzii & 1205 & 0,71 & 51 & 0 \\
Dabéma & Piptadeniastrum africanum & 1310 & 0,71 & 51 & 25 \\
Emien & Alstonia boonei & 1316 & 0,21 & 15 & 15 \\
Fraké / Limba & Terminalia superba & 1320 & 2,43 & 174 & 41 \\
Fromager/Ceiba & Ceiba pentandra & 1321 & 0.36 & 25 & 0 \\
Ilomba & Pycnanthus angolensis & 1324 & 0,71 & 51 & 0 \\
Iroko & Milicia excels & 1116 & 0,21 & 15 & 0 \\
Kotibé & Nesogordonia papaverifera & 1118 & 0.21 & 15 & 0 \\
Lotofa/Nkanang & Sterculia rhinopetala & 1212 & 0,36 & 25 & 0 \\
Movingui & Distemonanthus benthamianus & 1213 & 4,86 & 347 & 76 \\
Padouk rouge & Pterocarpus soyauxii & 1345 & 0,36 & 25 & 0 \\
Tali & Erythropleum ivorense & 1346 & 0.36 & 25 & 0 \\
\hline Total & & & $\mathbf{1 2 . 4 3}$ & $\mathbf{8 8 8}$ & $\mathbf{1 9 9}$ \\
\hline
\end{tabular}


REVUE DE L'ACADEMIE DES SCIENCES DU CAMEROUN Vol. 17 No. 1 (Octobre 2021)

Tableau 7. Table des stocks de l'assiette IRAD Oboul

\begin{tabular}{|c|c|c|c|c|c|}
\hline Essence & Nom scientifique & Code & Vol./ha & Vol. total & Vol. $\geq$ DME \\
\hline Aiélé / Abel & Canarium schweinfurthii & 1301 & 0,14 & 10 & 0 \\
\hline Aningré A & Aningeria altissima & 1201 & 2,59 & 185 & 185 \\
\hline Ayous / Obeche & Triplochyton scleroxylon & 1105 & 2,02 & 144 & 144 \\
\hline Bongo H (Olon) & Fagara heitzii & 1205 & 0,38 & 27 & 0 \\
\hline Dabéma & Piptadeniastrum africanum & 1310 & 1,83 & 131 & 121 \\
\hline Emien & Alstonia boonei & 1316 & 1,35 & 97 & 97 \\
\hline Fraké / Limba & Terminalia superba & 1320 & 3,8 & 272 & 140 \\
\hline Fromager / Ceiba & Ceiba pentandra & 1321 & 0,14 & 10 & 0 \\
\hline Ilomba & Pycnanthus angolensis & 1324 & 0,28 & 20 & 0 \\
\hline Iroko & Milicia excels & 1116 & 1,87 & 134 & 0 \\
\hline Kotibé & Nesogordonia papaverifera & 1118 & 0,19 & 13 & 0 \\
\hline Lotofa / Nkanang & Sterculia rhinopetala & 1212 & 0,17 & 12 & 0 \\
\hline Movingui & Distemonanthus benthamianus & 1213 & 8,1 & 578 & 291 \\
\hline Padouk rouge & Pterocarpus soyauxii & 1345 & 0,3 & 21 & 0 \\
\hline Tali & Erythropleum ivorense & 1346 & 0,3 & 21 & 0 \\
\hline Total & & & 23,46 & 1675 & 978 \\
\hline
\end{tabular}

Tableau 8. Variance du volume exploitable des essences principales par strate

\begin{tabular}{lllll}
\hline & Strate & $\begin{array}{l}\text { Nombre } \\
\text { de p.e. }\end{array}$ & $\begin{array}{l}\text { Variance } \\
\text { volume }\end{array}$ & $\begin{array}{l}\% \text { DME } \\
\text { à 95\% }\end{array}$ \\
\hline Secondaire & SJ & 5 & 63,042 & 74,66 \\
Sol hydromorphe & MIT & 1 & 0 & 0 \\
p.e $:$ Parcelles exploitables & & &
\end{tabular}

\section{Variance du volume exploitable}

Au regard de la contenance de la forêt étudiée, on a identifié deux écosystèmes : terres forestières $(83.13 \%)$ ) et terres non boisées $(16.87 \%)$ avec pour superficies respectives 71.41 ha et 14.49 ha (Tableau 8). Pour les terres boisées, mettre en place les parcelles de suivi permanente (4 parcelles) puis faire un marquage de chaque pied d'arbre (code spécifique) afin de faciliter un suivi permanent de chaque pied marqué.

Evaluation du potentiel non ligneux dans les zones d'étude

\section{Typologie des PFNL}

Le Tableau 9 fait état du type, les parties utilisées et l'importance des PFNL exploités dans la forêt étudiée. Il ressort de là que 18 PFNL sont utilisées dans la localité pour diverses importances à des fins purement subsistantes et pour la plupart récolté tout le long de l'année. Toutefois, les revenus tirés de cette activité demeurent faibles pour la très grande majorité de ces foyers (Lescuyer, 2010). Le Tableau 9 présente les 18 PFNLs présents dans la forêt étudiée et présente les différents usages et vertus propres à chacun d'entre eux. Il va jusqu'à montrer les parties utilisées par produit suivant le but recherché par l'utilisateur. De cette liste, six PFNLs s'utilisent 
fréquemment dans $65 \%$ des ménages enquêtés suivant une fréquences et méthode de collecte particulière. Cette utilisation régulière entraine un impact sur la biodiversité comme l'illustre le Tableau 10.

Tableau 9. Typologie des PFNL utilisées dans l'assiette IRAD Oboul

\begin{tabular}{|c|c|c|c|c|}
\hline $\mathrm{N}^{\circ}$ & Type & Nom scientifique & Partie utilisées & Importances/usages \\
\hline \multirow{3}{*}{1} & \multirow{3}{*}{ Ndiansang } & \multirow{3}{*}{ 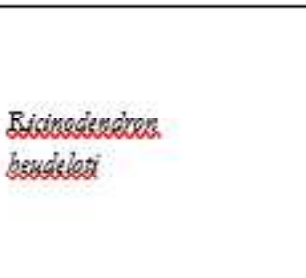 } & Graine & Alimentation \\
\hline & & & Capsules & $\begin{array}{l}\text { Feu de bois } \\
\text { Omement et décoration des maisons }\end{array}$ \\
\hline & & & Ecorce & $\begin{array}{l}\text { Santé (Stimule la production do lait } \\
\text { maternel) }\end{array}$ \\
\hline \multirow{2}{*}{2} & \multirow{2}{*}{ Xoacanga } & \multirow{2}{*}{ Talemerementara crassa } & Fruit & Santé (soigner les blessures graves) \\
\hline & & & Racines & Santé (mal de dents) \\
\hline 3 & $\begin{array}{l}\text { Emien/ } \\
\text { Elsouk }\end{array}$ & $\begin{array}{l}\text { Alstonia } \\
\text { kastrei }\end{array}$ & Ecorce & Santé (contre le paludisme) \\
\hline \multirow{6}{*}{4} & \multirow{6}{*}{ Palmier sauvage } & \multirow{6}{*}{$\begin{array}{l}\text { Elaik } \\
\text { suiesursis }\end{array}$} & Coton du palmier & Santé (en cas de brulures) \\
\hline & & & Fleurs & Santé (contre le mal de tête) \\
\hline & & & Palmistes & $\begin{array}{l}\text { Alimentation (fabrication de l'huile de } \\
\text { palme) }\end{array}$ \\
\hline & & & Noix & $\begin{array}{l}\text { Alimentation (fabrication de lhuile de } \\
\text { palme) }\end{array}$ \\
\hline & & & Vin blane & $\begin{array}{l}\text { Alimentation (fabrication de boisson } \\
\text { alcoolique local) }\end{array}$ \\
\hline & & & Rameaus & Artisanat \\
\hline \multirow{4}{*}{5} & \multirow{4}{*}{ Parasolier } & \multirow{4}{*}{$\begin{array}{l}\text { Mescasera } \\
\text { sercosiecies }\end{array}$} & Racines & Alimentation (collection de l'eau) \\
\hline & & & Bourgeons & Santé (brûlures) \\
\hline & & & Graines & Agriculture (comme biofertilisant du sols) \\
\hline & & & Bois & Utiliser pour la fabrication des pirogues \\
\hline 6 & $\begin{array}{l}\text { Afoambe jaune/ } \\
\text { Dffoile }\end{array}$ & $\begin{array}{l}\text { Enavtia } \\
\text { cblarethas }\end{array}$ & Ecorce & Santé (contre la typhoide) \\
\hline 7 & Dabema/Atas & 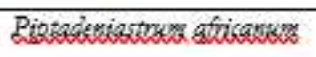 & Ecorce & Santé (contre le mal de reins) \\
\hline \multirow{2}{*}{8} & \multirow{2}{*}{ Mango/Adg'a } & \multirow{2}{*}{ Irvivgis agberreris. } & Pulpe & Alimentation \\
\hline & & & Noyau & Alimentation \\
\hline \multirow[t]{2}{*}{9} & \multirow[t]{2}{*}{ Tech } & \multirow[t]{2}{*}{ Tectena grandis } & Ecorce & $\begin{array}{l}\text { Santé (Stimule la production de lait chez } \\
\text { les jeunes mères) }\end{array}$ \\
\hline & & & Fevilles & Conception des nattes \\
\hline 10 & Raphia & Rapbia & Moelle & Utilisé pour la conception des maisons \\
\hline
\end{tabular}




\begin{tabular}{|c|c|c|c|c|}
\hline & \multirow{2}{*}{\multicolumn{2}{|c|}{ farieifers }} & \\
\hline & & & Vers blanc & Alimentation \\
\hline & & & Graines & Décoration des maisons \\
\hline & & & Tiges de raphiz & Artisanat \\
\hline 11 & $\begin{array}{l}\text { Afubala } \\
\text { Aldualaka }\end{array}$ & Pertachicra magrepledlla & Frust & Santé (huile corporelle) \\
\hline 12 & Colatier savvage & Cola viticis & Cola & Alimentation \\
\hline 13 & Safoutier sauvage & $\begin{array}{l}\text { Dacrovites } \\
\text { cuacreakilas }\end{array}$ & Fruit & Alimentation \\
\hline \multirow[t]{2}{*}{14} & \multirow[t]{2}{*}{ Doussié } & \multirow[t]{2}{*}{ Afralias 82} & Graines & $\begin{array}{l}\text { Santé (contre "limpuissance" chez } \\
\text { l'homme) }\end{array}$ \\
\hline & & & Ecorces & Santé (contre le mal de rein) \\
\hline \multirow[t]{2}{*}{15} & \multirow[t]{2}{*}{ Fromager } & \multirow{2}{*}{$\begin{array}{l}\text { Ceikg } \\
\text { Bertacka }\end{array}$} & \multirow[t]{2}{*}{ Fevilles } & Santé (contre le mal de dos) \\
\hline & & & & Alimentation \\
\hline 16 & Rotin & 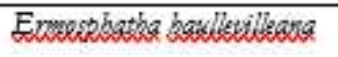 & Tige & Artisanat \\
\hline 17 & Bongo $\mathrm{H}$ & Fesarabekrii & Fruit & Alimentation \\
\hline 18 & Moabi & Bailerrerlla taxisecners & Fruit & Alimentation \\
\hline
\end{tabular}

Tableau 10. Caractérisation des PFNL les plus utilisés de l'assiette IRAD Oboul

\begin{tabular}{|c|c|c|c|c|c|c|}
\hline $\begin{array}{l}\text { Noms scientifiques } \\
\text { des essences }\end{array}$ & $\begin{array}{l}\text { Partie } \\
\text { utilisée }\end{array}$ & Provenances & $\begin{array}{l}\text { Méthode de } \\
\text { collecte }\end{array}$ & $\begin{array}{l}\text { Péniode de } \\
\text { collecte }\end{array}$ & $\begin{array}{l}\text { Impact sur } \\
\text { la ressource }\end{array}$ & But \\
\hline Baillarels sworsearess & \multirow{3}{*}{$\begin{array}{l}\text { Graines et } \\
\text { noyau }\end{array}$} & \multirow{3}{*}{ Grands arbres } & \multirow{3}{*}{$\begin{array}{l}\text { Ramassage } \\
\text { des fruits }\end{array}$} & \multirow{3}{*}{ Saisonnière } & \multirow{3}{*}{$\begin{array}{l}\text { Très } \\
\text { ellevé }\end{array}$} & \multirow{3}{*}{$\begin{array}{l}\text { Subsistance, } \\
\text { commercial }\end{array}$} \\
\hline 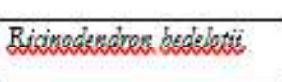 & & & & & & \\
\hline Indikgia s22, & & & & & & \\
\hline $\begin{array}{l}\text { Gancinsia teota, } \\
\text { Trichessugha } 128\end{array}$ & Pulpe & $\begin{array}{l}\text { Jeunes } \\
\text { arbres }\end{array}$ & $\begin{array}{l}\text { Cueillette, } \\
\text { ramassage }\end{array}$ & Saisonnière & Très faible & Subsistance \\
\hline Dacziodss macrothils & Pulpe & Grand axbre & Cueillette & Saisonnière & Très élevé & Subsistance \\
\hline
\end{tabular}




\section{Degré d'importance des PFNL Les fruits}

L'exploitation de fruits est largement influencée par la taille des arbres qui les produisent. Le Tableau 10 indique l'exploitation fruitière des jeunes arbres pour un but purement subsistant, provoque peu de dégâts au sein de la structure forestière. Par ailleurs, les espèces cibles pour la cueillette sont caractérisées par leur abondance, répartition sur l'étendue du territoire, disponibilité le long de l'année et leur saveur. Similaire observations ont été faites par d'autres études (Lescuyer, 2010; Tonga Ketchatang et al., 2017). Lorsque les arbres deviennent plus grands et inaccessible à la cueillette, la chute des fruits ou l'abattage de l'arbre reste les deux techniques d'exploitation. Cette technique non durable à des impacts similaires à l'exploitation abusive des PFL car elle cause la raréfaction des PFNL médicinaux (Zima et al., 2018). La deuxième technique entraine un fort dégât sur la forêt car favorise la destruction de la biodiversité forestière. Toutefois, une autre pratique consiste à couper l'arbre à hauteur du pied avant l'exploitation (Guedje et al., 1998). Pour d'autres essences, les fruits issus des arbres tombent et peuvent séjourner pendant une période donnée au sol avant d'être exploiter. C'est le cas des graines de Ndjansang (Ricinidendron heudelotii) et les graines de Moabi (Baillonela toxisperma).

\section{Les feuilles}

Les feuilles de certains arbres et arbustes sont utilisées comme légumes à l'Est Cameroun en général et dans le Haut-Nyong en particulier (Baobab) alors que certaines sont utilisées pour la pharmacopée traditionnelle (Adjanohoun et al., 1996). D'après Tchatat and Ndoye (2006), et la méthode d'exploitation utilisée lors de la collecte et son impact sur la ressource varient selon les espèces. Les pratiques de récolte des feuilles tendent à éliminer les plus grands individus reproducteurs à cause de leur abondance ce qui peut entrainer une baisse future du taux de régénération (Fedoung et al., 2021; Guedje et al., 1998).

\section{Ecorce, racines et tiges}

Elles sont la partie des essences la plus prisée dans la pharmacopée (Emien, Dabema et Doussié...). Plusieurs association et compositions sont faites par les herboristes du village afin de trouver des solutions spécifiques pour la guérison de différents maux. L'exploitation des écorces d'arbre a très souvent un impact très élevé sur la biodiversité car une surexploitation des écorces des essences expose l'aubier aux attaques des ravageurs. Ce qui fragilise le fonctionnement biologique de l'arbre et peut provoquer leurs chutes d'arbres en saison des pluies. En général, la commercialisation tant nationale qu'internationale représente la plus grande menace des PFNL car elle entraine la collecte abusive. Certaines écorces sont utilisées fabriquer le principe actif en laboratoire (Tchatat and Ndoye, 2006).

\section{Conclusion}

Il ressort de cette étude que les PFNL utilisés par les communautés d'Oboul sont à usage multiples et participent à la lutte contre la pauvreté via l'amélioration du bien-être de cette communauté. Les échanges engagés avec les principaux acteurs nous ont permis de déduire que le véritable goulot d'étranglement à la gestion durable des PFNL et PFL se traduisent parfois par une absence de convention de cogestion entre les centres de recherche (IRAD). Dans l'attente d'une éventuelle loi forestière spécifique aux PFN, nous pensons qu'il serait important de mettre en place des parcelles de suivi permanente nécessaire pour déjà circonscrire/localiser les pôles de production et faire un inventaire multi-ressources dans chacune des parcelles. Ce permettra non seulement pour la recherche mais aussi pour une gestion plus durable de nos ressources forestières (PFL et PFNL). 


\section{Bibliographie}

Adjanohoun, J. et al., 1996. Traditional medicine and pharmacopoeia. Contribution to ethnobotanical and floristic studies in Cameroon. Lagos, Nigeria: Organisation of African Unity. Scientific, Technical and research commission (OAU/STRC).

Eba, A., Lescuyer, G., Gouhouo, P., Moulende, F., 2013. Etude de l'importance économique et sociale du secteur forestier et faunique dans les Etats d'Afrique Centrale: Cas du Cameroun. Rapport d'étude CIFOR.

FAO, 2011. Rapport sur les forêts 2011 : La situation des forêts dans le bassin amazonien, le bassin du Congo et l'Asie du Sud-Est, Brazzaville. The Food and Agriculture Organization (FAO).

Fedoung, E.F. et al., 2021. A review of Cameroonian medicinal plants with potentials for the management of the COVID-19 pandemic. Advances in Traditional Medicine, 1-26.

Guedje, N.M., Van Dijk, H., Nkongmeneck, B.A., 1998. Ecologie et exploitation de quelques produits forestiers non ligneux (PFNL) de la forêt humide du sud-Cameroun. Séminaire FORAFRI, Libreville CIRAD.

Lescuyer, G., 2010. Importance économique des produits forestiers non ligneux dans quelques villages du Sud-Cameroun. BOIS \& FORÊTS DES TROPIQUES, 304, 15-24.

Moumbock, E.M.A., Ngaba, M.J.Y., ANGWAFO, T.E., 2020. Gestion durable des Hautes Valeurs de Conservation de types 5 et 6 dans les UFA gérées par la société forestière Pallisco : Identification, cartographie et enjeux pour la conservation. Hautes Valeurs de Conservation de types 5 et 6 .
Ngaba, M.J.Y. et al., 2019. Etude de faisabilité de la mise en place d'un site écotouristique dans le parc national de Boumba-Bek: cas des clairières forestières de Pondo. International Journal of Biological and Chemical Sciences, 13, 3177-3192.

Ngijol, R.D.B., Moumbock, E.M.A., Ngaba, M.J.Y., 2020. Contribution à l'élaboration d'un plan simple de gestion d'une forêt communautaire: Cas de l'inventaire d'aménagement de la forêt d'Alati (Sud-Cameroun). Journal of the Cameroon Academy of Sciences, 16, 53-69.

Olivier, I.M. et al., 2020. Suivi écologique de la dynamique des grands et moyens mammifères dans les clairières du parc national de Boumba Bek: cas du complexe de clairières de Pondo. Journal of Applied Biosciences, 144, 14755-14763.

Tchatat, M., Ndoye, O., 2006. Etude des produits forestiers non ligneux d'Afrique Centrale: reality and prospects. BOIS \& FORETS DES TROPIQUES, 289, 27-39.

Tonga Ketchatang, P., Zapfack, L., Kabelong Banoho, L.-P.-R., Endamana, D., 2017. Disponibilité des produits forestiers non ligneux fondamentaux à la périphérie du Parc national de Lobeke. VertigO-la revue électronique en sciences de l'environnement, 17.

Topa, G., Karsenty, A., Megevand, C., Debroux, L., 2009. Forêts tropicales humides du Cameroun: une décennie de réformes. The World Bank.

Zima, G.G., Mialoundama, F., Yangakola, J.M., Kossa, I., 2018. Importance Des Produits Forestiers Non Ligneux Medicinaux D'origine Vegetale Et Impacts Des Activites Anthropiques Sur Leur Durabilite Dans Le Sud-Ouest De La Republique Centrafricaine. 